Revue d'histoire de l'Amérique française

REVUE D.HISTOIRE DE L'AMÉRIQUE FRANÇAISE

\title{
Les députés conservateurs-nationalistes et le gouvernement Borden
}

\section{Le cas d'Albert Sévigny (1911-1913)}

\section{Réal Bélanger}

Volume 35, numéro 1, juin 1981

URI : https://id.erudit.org/iderudit/303926ar

DOI : https://doi.org/10.7202/303926ar

Aller au sommaire du numéro

Éditeur(s)

Institut d'histoire de l'Amérique française

ISSN

0035-2357 (imprimé)

1492-1383 (numérique)

Découvrir la revue

Citer cet article

Bélanger, R. (1981). Les députés conservateurs-nationalistes et le gouvernement Borden : le cas d'Albert Sévigny (1911-1913). Revue d'histoire de l'Amérique française, 35(1), 33-54. https://doi.org/10.7202/303926ar d'utilisation que vous pouvez consulter en ligne.

https://apropos.erudit.org/fr/usagers/politique-dutilisation/ 


\title{
LES DÉPUTÉS CONSERVATEURS- NATIONALISTES ET LE GOUVERNEMENT BORDEN - LE CAS D'ALBERT SÉVIGNY (1911-1913) -
}

\author{
RÉAL BÉLANGER* \\ Département d'histoire \\ Université Laval
}

La vague conservatrice qui balaie le gouvernement libéral de sir Wilfrid Laurier lors de l'élection générale du 21 septembre 1911, pour le remplacer par celui de Robert Laird Borden, envoie à Ottawa 27 députés conservateurs du Québec dont 17 conservateurs-nationalistes ${ }^{1}$. La présence à la Chambre des communes de ces conservateurs-nationalistes et surtout leurs relations futures avec le nouveau gouvernement suscitent alors le plus vif intérêt. La raison en est simple. Depuis 1910, des prises de position avaient si radicalement éloigné les conservateurs-nationalistes de leurs collègues tories qu'elles avaient même menacé le système de partis canadien à bipartisme rigide récemment mis au point par les chefs des deux formations politiques traditionnelles.

Cette scission émergea d'un conflit au sein de l'élite parlementaire du parti conservateur sur la question de la création d'une marine de guerre proposée par le gouvernement Laurier, le 12 janvier $1910^{2}$. L'aile québécoise canadienne-française, bien qu'unie aux autres conservateurs dans l'opposition au projet de loi, refusa d'entériner la position officielle du parti qu'elle jugeait insuffisamment canadienne et s'en sépara avec fracas. Ainsi isolée en Chambre et faible en nombre, elle y fut facilement vaincue. Son chef, Frédérick Debartzch Monk, député de Jacques-Cartier, décida aussitôt de porter les débats à l'extérieur du Parlement. Il convainquit l'opposition extra-parlementaire québécoise, représentée alors par le fougueux Henri Bourassa et son mouvement nationaliste, de

* L'auteur tient à remercier ses collègues Richard Jones, Jacques Mathieu et Yves Roby pour leurs précieux conseils.

1 Le Devoir, 19 août 1913, 1. Dans cet article, les termes «les tories», «le groupe Borden», se réfèrent au parti conservateur fédéral officiel. Le terme "conservateursnationalistes» désigne les conservateurs québécois liés aux nationalistes de Henri Bourassa.

2 Rappelons que ce projet de loi visait à créer une marine qui serait canadienne en temps de paix, mais britannique en temps de guerre. 
former une coalition qui s'exprimerait dans une série d'assemblées organisées dans tout le Québec. Ainsi naquit l'alliance conservatrice-nationaliste ${ }^{3}$.

Ce regroupement de l'opposition québécoise au projet de loi prit par la suite une ampleur remarquable. D'abord, les conservateurs de Monk, appelés désormais conservateurs-nationalistes ou encore «autonomistes», se rallièrent d'emblée aux principes fondamentaux du mouvement nationaliste canadien de Henri Bourassa. Ils appuyaient non seulement l'idée du plébiscite sur la question navale mais aussi celles de l'autonomie canadienne face à l'Angleterre et de la préservation de l'unité du pays par le respect de sa dualité. En outre, à l'instar des nationalistes, ils prônaient la liberté d'opinion si contraire à l'esprit du système de partis. Le groupe de Monk annonça même qu'il voulait former une équipe «de députés indépendants [capables de] faire échec à la loi navale et à toute autre mesure de contribution aux armements impériaux $[\ldots] \gg{ }^{4}$. Puis on passa à l'action. Les conservateurs-nationalistes mobilisèrent la grande majorité des conservateurs québécois, ratissèrent la province d'assemblées en compagnie de Bourassa et de ses disciples et combattirent ardemment lors des élections générales de 1911. Même s'ils contractèrent une alliance électorale assez scabreuse avec la très impérialiste formation de Borden, Monk et son équipe s'organisèrent alors «avec des quartiers généraux, des comités et tout un mécanisme entièrement distinct du parti conservateur ${ }^{5}{ }^{5}$. Au soir du 21 septembre 1911, 17 de ces conservateurs-nationalistes étaient élus mais sans parvenir à décrocher la balance du pouvoir, seul moyen valable pour que l'on tienne compte réellement de leur position à la Chambre des communes. Parmi eux, on retrouve Albert Sévigny, un jeune avocat de Québec, qui s'était pleinement identifié à ce groupe et n'avait rien ménagé pour se faire élire dans la circonscription de Dorchester.

Le vif intérêt soulevé au lendemain des élections autour des relations entre les conservateurs-nationalistes du Québec et le nouveau gouvernement Borden se comprend dès lors mieux. Ces députés «indépendants» du Québec, qui ne détiennent pas la balance du pouvoir, resteront-ils fidèles à leurs principes, à leurs actions antérieures, et continueront-ils à défier le système de partis canadien? La victoire du parti de Borden les conduira-t-elle à prolonger l'al-

3 Pour plus de précisions, voir Réal Bélanger, Albert Sévigny et le parti conservateur, thèse de Ph.D. (Université Laval, 1979), 75-84. Il subsistait des conservateurs irréductibles, fidèles à Borden.

4 Le Devoir, 29 mai 1913, 1.

5 Ibid., 30 mai 1913, 1; pour plus de précisions, voir Réal Bélanger, op. cit., 84-132. Notons que ni Henri Bourassa, ni Armand Lavergne, son disciple, ne se sont présentés lors de ces élections. 
liance électorale en une alliance parlementaire voire même gouvernementale avec ces tories? Parce que trop diversifiées, nous ne pouvons, dans le cadre de cet article, considérer toutes les facettes de l'évolution de ces relations. Nous avons plutôt choisi d'étudier un cas, celui d'Albert Sévigny, au cours des premières années de sa vie parlementaire à Ottawa. Ses prises de position illustrent bien l'attitude de ce groupe parlementaire que le mouvement nationaliste de Henri Bourassa jugera la plus dégradante.

\section{1 - Un appui conditionnel: 1911-1912}

Le nouveau député de Dorchester qui se présente à Ottawa après le 21 septembre 1911 n'a aucune expérience pratique du milieu parlementaire. Connu des cercles conservateurs québécois auxquels il s'était joint dès 1902 et auprès desquels il a déjà représenté une jeunesse agissante qui n'a pas craint d'affronter les anciens du parti afin de rénover une formation moribonde, il est cependant inconnu ou presque à Ottawa ${ }^{6}$. À l'aurore de la trentaine, ce jeune avocat ambitieux, promis à un bel avenir, selon le journaliste Georges Pelletier du Devoir, a encore beaucoup à apprendre et doit faire ses preuves?

Albert Sévigny est aussi un conservateur-nationaliste de la meilleure fournée. Depuis 1910, en effet, il s'est évertué à critiquer la marine Laurier, à pourfendre «la guenille bleue» qui sacrifiait l'autonomie canadienne au profit de l'impérialiste GrandeBretagne, à reconnaître Monk et Bourassa comme seuls chefs, à convaincre le Québec de ne pas s'occuper «de l'étroite question de parti» et d'envoyer à Ottawa 25 députés "indépendants»" ${ }^{8}$. Avec Armand Lavergne, il a su représenter le groupe conservateurnationaliste dans la région de Québec au même titre que Monk et Bourassa l'ont fait dans la région de Montréal. Aux élections de 1911 , son engagement en ce sens ne faisait aucun doute ${ }^{9}$. Rendu à Ottawa, confronté à un système de partis bien articulé, confronté, en somme, à la réalité complexe et nuancée de l'activité parlementaire, que fera-t-il?

Au cours de la première année de son mandat de député, on peut affirmer que, dans l'ensemble, Albert Sévigny respecte ses engagements nationalistes. Le tout, cependant, débute sur une note d'ambiguïté car, dans les premiers mois, le député de Dor-

6 Pour cette période de sa carrière, voir Réal Bélanger, op. cit., 16-132; il s'était aussi présenté dans la circonscription de Nicolet lors d'une élection partielle provinciale en 1907: il y fut défait.

7 Il est né le 30 décembre 1880; Le Devoir, 21 novembre 1911, 1.

8 Réal Bélanger, op. cit., 86, 101, 114.

9 La Patrie, 30 août 1911, 9. 
chester semble se rallier complètement au parti conservateur de Borden. C'est d'abord la formation du Cabinet qui en transmet timidement les premiers échos. Sévigny, le nationaliste, le candidat qui se disait indépendant de Borden, convoiterait un poste de ministre au sein du nouveau Cabinet ${ }^{10}$. Même si les libéraux dénoncent vivement cette ambition comme une compromission, il faut admettre que cet espoir ne l'oblige pas, en soi, à rejeter ses principes nationalistes ${ }^{11}$. Henri Bourassa lui-même n'accepte-t-il pas que Monk et deux autres députés conservateurs-nationalistes fassent partie de ce gouvernement transformant ainsi l'alliance électorale en alliance gouvernementale ${ }^{12}$ ? C'est sans doute là, malgré le risque évident d'absorption, le moyen de contrecarrer l'insuffisance de députés et de démontrer la force de ce groupe conservateur-nationaliste. En dépit de cela, il faut convenir que cette attitude de Sévigny demeure significative car elle révèle déjà les rapprochements auxquels il est prêt à consentir et dont le milieu politique devra tenir compte. Quant à ses ambitions personnelles, elles sont prématurées. Sans expérience de la vie parlementaire, ne possédant aucun «titre» valable, il ne fait pas le poids face à certains de ses collègues. Son nom, d'ailleurs, n'est même pas considéré dans la liste que font parvenir à Borden des conservateurs comme C.H. Cahan et William Price ${ }^{13}$. Le député de Dorchester doit donc attendre. Entre-temps, il choisit de raffermir ses liens avec le parti de Borden, choix, évidemment, qui ne fait qu'accentuer l'ambiguïté.

C'est à la Jeune-Lorette, le 27 octobre suivant, à l'occasion d'une assemblée en l'honneur du nouveau ministre des Postes, Louis-Philippe Pelletier, que Sévigny en dévoile le plus à cet égard. En effet, après avoir glorifié Bourassa comme «le plus grand

10 Robert Rumilly, Histoire de la province de Québec (Montréal, Éditions Bernard Valiquette, s.d.), XVI: 127; Le Soleil, 4 octobre 1911, 1.

11 Ibid.; Le Soleil, 10 octobre 1911, 4.

12 Monk obtient les Travaux publics, Wilfrid-Bruno Nantel, le Revenu de l'Intérieur et Louis-Philippe Pelletier, les Postes. Mème si Borden se garde le choix final, on peut dire qu'il abandonne virtuellement à Monk le choix de ses collègues francophones du Québec. Monk, conseillé par Henri Bourassa et Armand Lavergne, choisit des conservateursnationalistes au grand dam des autres conservateurs francophones moins liés à ce groupe. Henri Bourassa laissera par la suite entendre que les ministres conservateurs-nationalistes sont entrés dans le Cabinet à la condition que Borden respecte leurs principes: Le Devoir, 4 juin 1913,1; les recherches à ce sujet ne le confirment cependant pas: voir, entre autres, Roger Graham, "The Cabinet of 1911», dans Frederick W. Gibson, ed., Cabinet Formation and Bicultural Relations. Seven Case Studies [Ottawa], [Queen's Printer for Canada], [1970], 47-62; ou encore Heath N. Macquarrie, "Formation of Borden's First Cabinet», Canadian Journal of Economics and Political Science, XXIII, 1 (February, 1957): 90-104; ou, enfin, Robert Craig Brown, Robert Laird Borden, A Biography, I: 1854-1914, (Toronto, Macmillan of Canada, [c1975]): 206-211; les autres ministres du Québec sont Charles Joseph Doherty, à la Justice, et George Halsey Perley, sans portefeuille.

13 APC, Fonds Borden, Cahan à Borden, ler octobre 1911, MG26H, vol. 152: 81819-81821; Ibid., Price à Borden, 2 octobre 1911, MG26H, vol. 6: 640-642. 
canadien-français [sic] que nous possédions» et rappelé qu'il a parcouru la province en compagnie du chef nationaliste et de Lavergne, Sévigny déclare péremptoirement dans son discours qu'il n'est pas «nationaliste» et que Borden est désormais son seul chef $^{14}$. D'un coup, le député de Dorchester semble renier tous ses faits, gestes et paroles de la dernière année. Il serait redevenu, en somme, conservateur «orthodoxe» avec comme seul chef Borden. Même si l'on ne sait pas encore si le jeune avocat reviendra complètement et sans discernement à son parti ou s'il préférera plutôt la voie plus critique à laquelle il a habitué ses auditeurs, il y a lieu de s'interroger. Le pouvoir l'attirerait-il au point de l'amener à modifier son opinion? Et qu'en était-il de sa sincérité en 19101911? Si l'on ajoute à cela qu'à l'instar de tous ses collègues québécois sauf un, il s'inscrit comme conservateur dans le Canadian Parliamentary Guide, puis assiste aux caucus du parti, accepte d'appuyer l'adresse en réponse au discours du Trône et va même, dans ses premiers mois à la Chambre, jusqu'à donner son appui à toutes les politiques mises de l'avant par le gouvernement Borden, toutes les spéculations sont possibles ${ }^{\text {. }}$.

Il n'en faut pas davantage pour soulever la colère de Laurier et de la presse libérale du Québec. Dans un discours mordant qu'il prononce au Monument national de Montréal, le 8 janvier 1912, le chef libéral fustige ces «anciens nationalistes» qui suivent en tous points les directives de Borden et qui demeurent cois sur la question de la marine oubliée jusqu'alors par le chef conservateur. Dans un trait d'esprit mi-moqueur, mi-incisif, Laurier ramène le mouvement nationaliste à des limites côtoyant la vénalité: «Le maître, clame-t-il, disait que le nationalisme était un mouvement; nous l'avons vu, c'était en effet un mouvement vers la crèche. $)^{16}$ Le Canada, le journal libéral de Montréal, renchérit en spécifiant nettement que «le nationalisme n'était qu'un masque pour tromper les électeurs» alors que Le Soleil, son confrère de Québec, relève que «ces braves bleus, castors et totonomistes, ont rengaîné

14 L'Événement, 28 octobre $1911,1,3$.

15 Ses collègues québécois appuient aussi inconditionnellement Borden; The Canadian Parliamentary Guide, 1912 (Ottawa, Montréal, Mortimer Company Limited, 1912), 185-186. Seul Adélard Bellemare, député de Maskinongé, s'inscrit comme conservateurindépendant: voir Ibid., 125. Il est bon de noter que le Canadian Parliamentary Guide est produit à partir des données des députés eux-mêmes; Heath Macquarries, The Conservative Party ([Toronto], McClelland and Steward Limited, [c 1965]), 79; L'Evénement, 23 octobre 1911,8 ; à noter aussi, qu'habituellement, le premier ministre choisit pour proposer et appuyer l'adresse en réponse au discours du Trône des députés voués à un avenir politique prometteur. C'est R.B. Bennett qui proposera l'adresse; Compte rendu officiel des Débats de la Chambre des Communes du Canada, session 1911-1912, vol. I: 27-34 (à l'avenir, nous n'utiliserons que les mots Les Débats).

16 Le Soleil, 9 janvier 1912, 9. 
toute la kyrielle de leurs litanies anti-marinières; ils s'assoient sur le plébiscite et rêvent du statu quo»" ${ }^{17}$. Pour les libéraux, Albert Sévigny et ses collègues francophones du Québec trahissent leurs paroles de 1910-1911: ils en concluent donc qu'il n'y a plus rien à attendre de ce groupe. Pourtant Henri Bourassa, celui qui les a conduits là où ils sont, leur fait encore confiance. Il attend, avant d'entreprendre toute critique, qu'une politique touchant vraiment aux principes nationalistes soit présentée en Chambre ${ }^{18}$. C'est alors que Sévigny, en particulier, révélera plus précisément le sens qu'il veut donner à la nouvelle orientation de sa carrière dans le parti conservateur et, surtout, démontrera son respect de la pensée nationaliste.

À ce dernier égard, Sévigny commence l'année 1912 sur le bon pied en s'attaquant directement à la loi navale de Laurier. Dès le 7 février, il présente à la Chambre un avis de motion qui traduit exactement la doctrine véhiculée par les conservateursnationalistes en 1910-1911. Il demande, en effet:
that [...] The Naval Service Act [...] should be repealed, because this Act establishes a line of conduct the importance and consequence of which are very great, and that the propos- ed Navy was not discussed before the people previous to being adopted; and that it carries all the marks of a plan tacitly pre- pared and badly elaborated; because our duty to the Empire cannot be properly fulfilled by putting this measure in force; and that this plan means the creation of a Naval force which will be absolutely useless in time of war and consequently of no benefit for Canada or the Empire; that it will require immense sums of money to build, equip and maintain this Navy; and the probable result is that in the time of war useful lives will be sacrified for nothing and without having added one iota to the fighting force of the Empire and of Canada. ${ }^{19}$

Borden, qui veut vraisemblablement se donner du temps dans cette importante question de la marine de guerre, refuse cependant la discussion car celle-ci, avance-t-il officiellement, «[retarderait] sérieusement les travaux de la Chambre» ${ }^{20}$. Sévigny ne peut que se soumettre mais, chose certaine, son action n'a été ni inutile ni seulement intéressée comme l'affirment alors Le Canada et Le

17 Le Canada, 1er décembre 1911, 1; Le Soleil, 18 novembre 1911, 4.

18 Le Devoir, 5 juin $1913,1$.

19 House of Commons, Routine Proceedings And Orders Of The Day For Tuesday, February 27, No 50 (Ottawa, Canada, 1912), 15: l'avis de motion est présenté le 7 février et porte le no 19.

20 Les Débats, session 1911-1912, vol. III: 4334. Nous discuterons plus à fond de l'attitude de Borden face à la question navale dans la partie II. 
Soleil $^{21}$. Outre le fait qu'elle oblige Borden à révéler que la loi navale ne sera pas révoquée lors de la première session de cette nouvelle législature, elle montre, en effet, un Sévigny prêt à poser un geste qui aurait pu rouvrir directement les débats autour de cette épineuse question. C'est là un geste conforme à la ligne de conduite conservatrice-nationaliste d'avant la prise du pouvoir.

Il en sera de même au moment de la question dite du «Keewatin» que Borden s'affaire alors à parachever. Cette question, délicate s'il en est une, parce que touchant directement à un principe nationaliste, occupe les esprits en ce début de l'année $1912^{22}$. Depuis la mi-janvier, en effet, les cercles ministériels discutent du partage des Territoires du Nord-Ouest entre l'Ontario, le Québec et le Manitoba. Le gouvernement conservateur du Manitoba, à la veille des élections, aimerait bien profiter de l'annexion d'une partie du district du Keewatin située au nord pour refaire son image électorale ternie par les scandales. De 73000 milles carrés, la superficie de la province atteindrait ainsi 251000 milles carrés avec un débouché sur la baie d'Hudson ${ }^{23}$. Or, une question cruciale vient se greffer à cette situation, celle du respect des droits scolaires de la minorité du Keewatin. Jusqu'à ce moment, le district avait été sous juridiction immédiate de l'autorité fédérale qui acceptait les écoles séparées dans les Territoires du Nord-Ouest par suite de l'article 11 de la loi fédérale de 1875. De fait, des écoles séparées avaient été créées mais le district n'avait pu se donner un système scolaire dûment constitué. Deux problèmes majeurs liés à l'annexion du Keewatin au Manitoba se posent donc en ce début de 1912. Puisqu'il n'y a pas de système scolaire établi comme tel au Keewatin, la minorité peut-elle revendiquer des droits scolaires acquis? Et, puisque, pour sa part, le Manitoba a aboli le type d'écoles séparées tel que défini dans la loi fédérale de 1875, la partie catholique des 165 Blancs, des 500 Métis et d'un certain nombre d'Indiens sera-t-elle privée, après l'annexion, du genre d'écoles séparées qu'elle connaît à ce moment ${ }^{24}$ ? Si cela se produit, elle risque l'extinction. Dans cette période d'incertitude, Mgr Adélard

21 Le Canada, 8 février 1912, 1; Le Soleil, 9 février 1912, 4. Ces deux journaux croient à un "Bluff» car par suite de règles parlementaires, la motion Sévigny ne peut être discutée qu'avec le consentement même du gouvernement: voir aussi Le Devoir, 7 février 1912,6 .

22 L'Action française, éd., Paul-Émile Lamarche. Oeuvres-Hommages (s.1., Bibliothèque de l'Action française, s.d.), 244. Cette question occupe cependant une place secondaire dans l'ensemble des problèmes politiques considérés alors par le gouvernement Borden.

23 La Patrie, 27 février 1912, 1.

24 Chiffres extraits du discours de Borden, Les Débats, session 1911-1912, vol. III: 4589; Abbé Lionel Groulx, L'Enseigrement français au Canada. Les Écoles des Minorités (s.1., Librairie Granger Frères, Limitée, [1933]), II: 138-145. 
Langevin, archevêque de Saint-Boniface, et Mgr Ovide Charlebois, vicaire apostolique du Keewatin, insistent auprès de Borden afin qu'il sauvegarde les droits de la minoritée ${ }^{25}$. Que fera donc le gouvernement Borden ainsi confronté pour la première fois à la présentation d'une politique concernant le respect des deux races du pays, principe de base du mouvement nationaliste de Henri Bourassa? Albert Sévigny, quant à lui, a effectué son choix: il opte pour la défense des droits de la minorité.

Mais la chose ne sera pas facile car le système de partis, si peu menacé depuis septembre 1911, met en branle tous ses atouts. L'affaire se jouant dans les coulisses, rumeurs et manoeuvres se succèdent à un rythme effarant. Le gouvernement, quelque peu hésitant semble-t-il, attend, écoute, consulte alors que les ministres québécois tiennent des réunions prolongées avec leurs députés ${ }^{26}$. Progressivement, vers la mi-février, la décision gouvernementale se dessine: une décision qui pourrait ne pas satisfaire la minorité27. Armand Lavergne, le toujours fidèle et principal disciple de Bourassa, accourt alors à Ottawa pour y apporter un message du maître qui exige, ni plus ni moins, qu'une clause garantisse les droits de la minorité ${ }^{28}$. Ill y découvre une députation conservatricenationaliste des plus décimées qui ne semble pas majoritairement partager ses vues. Déçu, il confiera plus tard:

Je me rendis à Ottawa: ce ne fut pas long avant que je ne fusse informé par l'honorable sénateur Landry, président du Sénat, que les «farouches nationalistes», comme on les appelait, semblaient être des gens de composition, de très bonne composition même.

À part une demi-douzaine, ayant à leur tête le sénateur Landry, Paul-Émile Lamarche [,] [...] Barrette [Sévigny, etc,], les autres paraissaient hésitants, mous ou indifférents. ${ }^{29}$

Malgré l'épine que représente un petit groupe de récalcitrants dont fait partie Albert Sévigny - maints commentaires de la presse parlent même de «crise» chez les députés conservateurs -, Borden a beau jeu ${ }^{30}$.

Le 27 février, il annonce officiellement en Chambre les modalités de l'annexion d'une partie du Keewatin au Manitoba. Le pro-

25 APC, Fonds Borden, Mgr Langevin à Borden, 29 janvier 1912, MG26H, vol. 134, 70989, 70990; Ibid., Mgr Charlebois à Borden, 31 janvier 1912, MG26H, vol. 134, 70992, 70993.

26 Le Devoir, 16 février 1912, 1.

27 L'Action française, éd., op. cit., 245.

28 La Patrie, 23 février 1912, 4; Ibid., 24 février 1912, 4.

29 Armand Lavergne, Trente ans de vie nationale (Montréal, les Éditions du Zodiaque, 1934), 213.

30 Le Devoir, 26 février 1912, 6. 
jet touche aux conditions matérielles mais, comme on l'avait anticipé, n'aborde pas la question scolaire ${ }^{31}$. Face à cette rebuffade, le petit groupe de rebelles et Albert Sévigny ne peuvent rester indifférents, mais le temps joue contre eux. Le projet de loi, voté en première lecture la journée même de sa présentation, ne doit être présenté en seconde lecture, l'étape des discussions véritables, que la semaine suivante. Entre-temps, le gouvernement met en oeuvre toutes sortes de «moyens» pour paralyser le petit groupe et, qui sait, le convertir peut-être. Robert Rogers, ministre de l'Intérieur, le «stratège par excellence» du parti, est chargé de cette mission.

Rogers entreprend des négociations «secrètes» afin d'éliminer toute opposition au projet ${ }^{32}$. Il rencontre les députés conservateurs canadiens-français et leur promet que Rodmond Roblin, le premier ministre manitobain, réglera l'affaire des droits de la minorité à la satisfaction des catholiques du Keewatin ${ }^{33}$. Si on lui demande un texte formel de garantie, il refuse allant même jusqu'à affirmer la détermination du gouvernement à passer outre à toute démission ${ }^{34}$. Tôt, aussi, les discussions s'engagent sur un autre terrain. On essaie de persuader les récalcitrants en leur faisant miroiter un bel avenir s'ils demeurent fidèles à la politique du parti. Des postes de ministres et de sous-secrétaires parlementaires rémunérés \$5000 par année, en sus de l'indemnité parlementaire, pourraient devenir accessibles ${ }^{35}$. Alors que les ministres québécois se laissent tous persuader, Albert Sévigny et le petit groupe, non sans peine cependant, s'opposent avec ténacité ${ }^{36}$.

La preuve en est donnée lors des discussions en deuxième et troisième lectures qui débutent le 4 mars. D'abord, dès le deuxième jour des débats, Sévigny, ainsi que quatre de ses collègues récalcitrants, appuient un amendement de Laurier qui, bien qu'équivoque, peut au moins rallier les adversaires du projet ministériel ${ }^{37}$. Par la suite, soit depuis cette soirée du 5 mars jusqu'au 12 mars, date du vote final, le député de Dorchester confirme, tant par ses

31 Les Débats, session 1911-1912, vol. II: 3981-3990; voir aussi Ramsay Cook, "Church, Schools, and Politics in Manitoba, 1903-1912», dans Ramsay Cook, Craig Brown, Carl Berger, ed., Minorities, Schools and Politics ([Toronto], University of Toronto Press, [c 1969]) (Coll. Canadian Historical Reading, no 7), 39-40.

32 Le Devoir, 28 février 1912, 6.

33 Ibid.; La Patrie, 2 mars 1912, 10.

34 L'Action française, éd., op. cit., 247; Robert Rumilly, Histoire de la province de Québec (Montréal, Montréal-Éditions, s.d.), XVII: 63.

35 Le cas de Paul-Emile Lamarche est sans doute révélateur de celui de ses collègues dont Sévigny: L'Action française, éd., op. cit., 246.

36 Robert Rumilly, op. cit., XVII: 63.

37 Les Débats, session 1911-1912, vol. III: 4470; Ibid., 4488-4490; ces quatre autres collègues sont Paul-Emile Lamarche, Adélard Bellemare, J-P-O. Guilbault et Eugène Paquet. À noter que Monk appuie le gouvernement. Pour lui, «il n'y a pas de droits [...] acquis dans le territoire [...]». Ibid., 4514. 
votes que par ses appuis moraux, son attitude sur l'amendement Laurier. Nul doute qu'il seconde, à chaque occasion, les efforts de Paul-Émile Lamarche qui, aidé de Montréal par Bourassa, dirige inlassablement les gestes du petit groupe ${ }^{38}$. Lors du vote en deuxième lecture, par exemple, Sévigny réitère, à la suite des quatre autres opposants, son désaccord avec le ministère, désaccord qu'il maintient en troisième lecture en soutenant, le 12 mars, l'amendement «Mondou-Lamarche» visant nettement à protéger les droits ou privilèges de la minorité ${ }^{39}$. À la veille de la clôture des débats, au moment de la discussion d'un amendement du libéral H.-S. Béland, il va même jusqu'à se prononcer verbalement sur la question alors qu'au-delà de ses griefs contre les arguments du député libéral, ses propos révèlent avec un accent d'émotion la force, le courage et, semble-t-il, les sacrifices que nécessite son engagement.

Pour ma part, clarifie-t-il, j'ai pris sur cette question une attitude bien sincère et franche; une attitude qui a exigé de moi un sacrifice considérable. Depuis dix ans que, pour mon malheur, je fais de la politique, j'ai toujours lutté pour le parti conservateur dans la province de Québec; j'ai pris part à toutes les luttes dans les plus mauvais jours du parti, et quelques mois après mon entrée dans cette Chambre, j'ai constaté que ma conscience m'obligeait à voter contre le parti pour lequel j'ai toujours combattu. Je le déclare, c'est un grand sacrifice; je l'ai fait pour harmoniser mon vote avec ma conscience passée, et rendre justice à ce que $\mathrm{ma}_{40}$ conscience me criait être mon devoir dans les circonstances. ${ }^{40}$

Jusqu'à la fin, donc, mais sans réussir à faire bouger le gouvernement, Sévigny reste solidaire du groupe de récalcitrants et s'oppose aux visées de Borden. Le député de Dorchester tourne alors l'une des pages dramatiques de sa jeune carrière.

Quelle interprétation peut-on donner à l'attitude de Sévigny sur la question du Keewatin? D'abord, Albert Sévigny démontre bien qu'il entend demeurer intraitable sur le principe fondamental du respect des deux races du pays. Puis, il révèle explicitement l'orientation qu'il privilégie au sein du parti conservateur: il acquiesce, en fait, généralement aux politiques du parti, mais il se garde la liberté de s'en dégager quand elles ne satisfont pas sa cons-

38 Robert Rumilly, Henri Bourassa. La vie publique d'un grand Canadien (Montréal, Les Éditions de l'homme, [1953]), 448-455; Ramsay Cook, loc. cit., 39.

39 Les Débats, session 1911-1912, vol. III: 4590-4592. À noter que le ministère emporte la deuxième lecture par 114 voix à 76 . Sur l'amendement Mondou-Lamarche, que le ministère défait par 160 voix contre 24 , le petit groupe se renforce de 2 membres, soit J-A. Barrette et A-A. Mondou.

40 Ibid., 5035. On remarque sans difficulté les lacunes de mémoire de Sévigny sur la période 1910-1911. 
cience et sa conduite passée. À l'encontre de la majorité des autres conservateurs-nationalistes, dont les prises de position illustrent bien l'échec principal de l'élection de 1911 qui les a isolés devant le bloc Tory, Albert Sévigny confirme, en somme, sa fidélité aux engagements des années 1910-1911.

Cependant, on se rend facilement compte que cette orientation semble difficile à accepter et à concrétiser. Sévigny ne va-t-il pas jusqu'à parler de sacrifices et dire que c'est pour son malheur qu'il fait de la politique depuis dix ans? C'est qu'élu, le jeune député de Dorchester est beaucoup plus conscient de tout ce que cette attitude peut lui coûter en «patronage» et en avancement personnel. Il sait que, noble en soi, cette position risque de lui faire subir les rudes coups des contraintes du milieu. Auparavant, il était facile de crier du haut des estrades qu'il fallait élire un groupe de députés indépendants qui sauraient faire respecter des principes à Ottawa. Maintenant, au contact de députés devenus ministres, couverts d'honneurs et de prestige, Sévigny s'interroge sur la portée ultime de chacun de ses gestes dans un système de partis qui pardonne peu. Sur cette question du Keewatin, toutefois, il relève élégamment le défi des honneurs et des principes et va même jusqu'à avouer à la Chambre qu'il le fera encore s'il le faut ${ }^{41}$. Au printemps de 1912, malgré des tiraillements indéniables, Sévigny refuse donc de céder.

\section{II - Retour au conservatisme: 1912-1913}

La deuxième moitié de l'année 1912 et l'année 1913 marquent un changement d'attitude notoire de la part d'Albert Sévigny envers le gouvernement Borden et le mouvement nationaliste de Henri Bourassa, changement d'attitude qui, de fait, modifiera l'orientation de la carrière politique du député de Dorchester. Au-delà de sa participation à l'élection provinciale de mai 1912, au cours de laquelle il s'efforce d'atténuer les remous causés par le comportement du gouvernement fédéral sur la controverse du Keewatin, c'est essentiellement sa prise de position sur l'épineuse question de la contribution canadienne à la défense navale de l'Empire, telle que Borden la conçoit à la fin de l'été de cette première année de pouvoir, qui provoque le tout ${ }^{42}$.

Cette importante affaire surgit précipitamment le 18 mars 1912 alors que des propos alarmants du premier lord de l'amirauté au sujet de la défense navale de l'Empire obligent Borden à entre-

41 Ibid., 5060.

42 Pour l'élection générale provinciale de 1912, voir L'Événement, 7 mai 1912, 4, 6 et Le Soleil, 7 mai 1912, 4, 8 mai 1912, 4, 14 mai 1912, 12. 
prendre rapidement les préparatifs d'un voyage à Londres afin de s'enquérir de l'urgence de la situation et du type de contribution recherchée par la mère patrie qui se sent menacée par l'Allemagne ${ }^{43}$. Cette conjoncture, cependant, dérange les projets du premier ministre qui devra ressusciter plus tôt qu'il ne l'aurait souhaité l'embarrassante question de la marine de guerre tant discutée en 1910-1911 et liée étroitement à ce débat. Sachant fort bien que les conservateurs-nationalistes et les impérialistes de son parti ne s'étaient entendus en 1911 que sur leur opposition au fameux projet Laurier, Borden avait décidé jusque-là de différer souvenons-nous de la motion Sévigny - l'abrogation de la loi de 1910 et de concevoir, le moment venu, c'est-à-dire après avoir été dûment informé par Londres, "a different permanent policy for naval defence, a policy which he hoped would have the support of both the Nationalists and the Imperialists of his party" ${ }^{44}$. Se rappelant un passé récent au cours duquel sa formation politique avait éclaté précisément sur cette affaire, Borden, prudent, avait donc voulu se donner du temps. Mais là, en cette fin de mars 1912, il n'a plus le choix: il doit courir le risque de provoquer les deux ailes radicales de son parti. À nouveau, les conservateurs-nationalistes et Albert Sévigny seront amenés à prendre de déchirantes décisions.

Déjà convaincu, semble-t-il, de la nécessité d'une aide concrète à la mère patrie, Borden part pour Londres en juin et en revient le 8 septembre "armed with two memoranda from the Admiralty, drafted by Churchill [et lui-même], to convince Parliament and [the] Cabinet [et plus particulièrement ses collègues canadiens-français] of the necessity of an emergency contribution" ${ }^{45}$. Commencent alors au pays, et plus particulièrement dans le cercle restreint du Cabinet, les vraies discussions autour du futur projet de loi tandis que les rumeurs les plus diverses circulent dans la presse et dans les milieux parlementaires ${ }^{46}$. Puis, le 18 octobre,

43 Face au nouveau programme de construction navale allemand, Winston Churchill, le premier lord de l'amirauté, invitera, le 18 mars, les Dominions à contribuer à la contre-partie britannique: voir Robert Craig Brown, Robert Laird Borden. A Biography..., 235-236.

44 Gilbert Norman Tucker, The Naval Service of Canada. Its Official History. Origins and Early Years (Ottawa, King's Printer, 1952), t. 1: 173; il faut dire cependant que Borden avait déjà promis - probablement dans le but de satisfaire les conservateursnationalistes - d'abroger la Loi Laurier: Les Débats, session 1911-1912, vol. I: 60-61 et Ibid., vol. III: 5467; Robert Craig Brown, op. cit., 235.

45 Heath Macquarrie, ed., Robert Laird Borden: His Memoirs (Toronto, Montréal McClelland and Stewart Limited, [c 1969]), t. 1: 165, (Coll. The Carleton Library, no 46); Robert Craig Brown, Ramsay Cook, Canada, 1896-1921. A Nation Transformed ([Toronto], McClelland and Stewart Limited, [c 1974]), 207; L.-P.Pelletier, J.D. Hazen et C.J. Doherty accompagnent le premier ministre. Monk a refusé de le suivre: Ibid., 205.

46 Voir, par exemple, Le Soleil, 26 septembre 1912, 4, 30 septembre 1912, 1 et Le Devoir, 1er octobre 1912, 1. 
coup de théâtre! Monk, le ministre québécois le plus surveillé sur cette question, renseigné par Borden sur la contribution projetée, remet sa démission ${ }^{47}$. Personne, désormais, ne peut croire que le projet de loi respecte les politiques défendues par les conservateurs-nationalistes du Québec en 1910-1911. Dans ces circonstances, comment réagira Albert Sévigny, l'ardent défenseur de l'autonomie canadienne des années 1910-1911?

Jusqu'à ce qu'il assure Borden de son appui, quelques indices avaient révélé aux observateurs la nouvelle orientation de Sévigny sur la coopération navale avec l'Empire. D'abord, il participe à la campagne électorale dans Richelieu en appuyant la candidature du conservateur Edward André D. Morgan alors que Monk avait déjà démissionné pour protester contre la politique navale de Borden ${ }^{48}$. $\mathrm{Sa}$ présence dans Richelieu, dans cette période d'attente, n'indique-t-elle pas que Sévigny délaissera Monk? Pour Le Soleil, il n'y a pas à en douter: «Sévigny [...] [a jeté], en fait, [son] bonnet nationaliste par-dessus les moulins [et endosse la] politique de contribution d'urgence sans recours au plébiscite. Il est impossible d'interpréter autrement [sa] descente dans le comté de Richelieu, au lendemain de la démission de M. Monk.» ${ }^{49}$ Puis, en novembre, Sévigny seconde les efforts de Louis Coderre qui tente de se faire réélire dans Hochelaga après avoir accepté de succéder à Monk dans le Cabinet Borden ${ }^{50}$. Cette fois, le député de Dorchester peut difficilement cacher qu'il entend entériner la nouvelle politique navale, dont on ignore encore officiellement la teneur. Cette fois

47 Monk admet alors l'urgence mais exige la consultation populaire par la voie du plébiscite. Borden, qui n'a pas ménagé les efforts afin de convaincre Monk de demeurer à son poste, croyait jusqu'alors qu'en démontrant sans équivoque l'état d'urgence, il rallierait à son projet ses collègues canadiens-français: APC, Fonds Borden, Privés, Journal personnel, 17 septembre 1912, 26 septembre 1912, 8 octobre 1912, 9 octobre 1912 , 10 octobre 1912; c'est le 14 octobre que Borden lit au Cabinet le futur projet de loi: Ibid., 14 octobre 1912; APC, Fonds Borden, Monk à Borden, 18 octobre 1912, MG26H, vol. 33: 13690; le 19 octobre, Pelletier et Nantel, les deux autres ministres francophones du Québec, disent à Borden qu'ils ne démissionneront pas: APC, Fonds Borden, Privés, Journal personnel, 19 octobre 1912 .

48 L'élection du libéral Pierre-Joseph-Arthur Cardin, le 21 septembre 1911, a été invalidée et il faut la recommencer. P-J-A. Cardin se présente à nouveau contre E.A.D. Morgan et la date du scrutin est fixée au 24 octobre 1912. Cardin l'emporte par 258 voix de majorité: James G. Foley, Rapport sur les Élections partielles (12e Parlement) pour la Chambre des communes du Canada tenues pendant l'année 1912 (Ottawa, C.H. Parmelee, Printer to the King's Most Excellent Majesty, 1913), 15. Cette victoire n'est pas une surprise pour Borden qui prévoyait une défaite pour son parti: APC, Fonds Borden, Privés, Journal personnel, 24 octobre 1912.

49 Le Soleil, 24 octobre 1912, 4.

50 Borden avait offert la succession de Monk à Tom Chase Casgrain et à L-T. Maréchal qui l'ont déclinée: voir APC, Fonds Borden, Privés, Journal personnel, 23 octobre 1912 et 26 octobre 1912. Louis Coderre devient Secrétaire d'Etat, Rogers accepte le portefeuille des Travaux publics et William James Roche, celui de l'Intérieur; la coutume veut qu'un député nommé ministre se représente devant ses électeurs. 
aussi, les nationalistes, et particulièrement Armand Lavergne, commencent à s'acharner contre lui.

Lors de cette élection, seuls les nationalistes, par leur candidat Léopold Doyon, s'opposent à Louis Coderre qu'ils accusent d'avoir trahi ses paroles des années 1910-1911 ${ }^{\text {s1 }}$. Une lutte des plus vives s'engage où la plupart des grandes personnalités des deux formations s'affrontent dans la presse ou sur les estrades: la rupture entre conservateurs et nationalistes prend alors une tournure violente $^{52}$. Albert Sévigny et Armand Lavergne, par exemple, s'échangent les pires insultes et s'accusent mutuellement. Pour Lavergne, Sévigny n'est plus qu'un «traître, un renégat, un créchard et un vendu». Sévigny réplique en fustigeant et répudiant le mouvement nationaliste et ses adeptes qu'il identifie maintenant, et à son tour, à des agitateurs et des gens qui ne respectent pas leurs adversaires:

Je n'ai jamais été insulté, crie-t-il, avant la semaine dernière et il a fallu que ce soit les Nationalistes qui m'insultent les premiers. On a dit que le député de Dorchester était un traître et un renégat. Nous sommes habitués à ces injures dans la province de Québec. [Oubliant ses paroles de 1910, il ajoute] depuis quelques années, les Nationalistes les ont jetées à la face d'un des plus grands Canadiens, [sir Wilfrid Laurier] [...], aujourd'hui, c'est notre tour. C'est M. Armand Lavergne qui nous a injurié depuis le commencement de la campagne [...]. [Mais] cet homme qui croit avoir le monopole de la vertu, qui pose au sauveur de la patrie n'est pas indifférent devant la crèche $[\mathrm{car}][\ldots]$ il a demandé un portefeuille dans le Cabinet Borden, [...] il a sollicité du gouvernement la place d'avocat-conseil de la Commission du Havre de Québec [et] a sollicité des ministres de gros contrats pour ses amis. ${ }^{53}$

Les deux amis des années 1910-1911, qui ont bravé ensemble les foules, se brouillent et se lanceront longtemps encore les récriminations les plus acerbes: entre eux, le duel à finir est commencé.

Cette campagne d'Hochelaga, que les nationalistes concentrent autour du futur projet de la marine Borden et de la démission de Monk, amène nécessairement les conservateurs présents à se prononcer sur cette épineuse question ${ }^{54}$. Mais ils évitent de prendre une position définitive, même si leur participation à la lutte les

51 Laurier, par stratégie, refuse toute candidature libérale. Les nationalistes parleront alors de collusion entre les deux partis traditionnels. En fait, sans être liés aux conservateurs, les libéraux leur accorderaient leur appui pour écraser davantage ce qui reste du mouvement nationaliste.

52 Robert Rumilly, Henri Bourassa. La vie publique..., 467.

53 L'Événement, 19 novembre 1912, 8.

54 Le Devoir, 13 novembre 1912, 1. 
identifie nettement. Sévigny, pour sa part, déclare qu' «il attend l'énoncé de la politique navale avant de la juger, puis il dénonce [...] les agitateurs qui veulent soulever le peuple avant de connaître la situation. ${ }^{55}$ Le Soleil, à nouveau, le rive à la réalité:
Parmi les comédiens de la Grande Farce [de] l'année dernière [...], il n'en fut pas de plus ardent, de plus enflammé, de plus truculent, de plus tonitruant que M. Albert Sévigny! [Mais], ce capitaine de l'armée du salut bourrassiste, devenu aphone, est passé armes et bagages, dans le camp tory-impérialiste, et complice complaisant, endosse de son mutisme, les trahisons de son chef de file, Louis-Philippe Pelletier. ${ }^{56}$

En somme, et malgré les dénégations du député de Dorchester, la campagne d'Hochelaga, facilement remportée par Coderre, confirme aux observateurs ce que celle de Richelieu avait révélé: Albert Sévigny est à la veille de modifier officiellement son opinion sur la coopération navale du Canada avec l'Empire ${ }^{57}$. Et ils ne se trompent pas.

Lorsque le gouverneur général procède à la lecture du discours du Trône de la deuxième session du douzième Parlement devant les deux Chambres réunies dans la salle des séances du Sénat, le 21 novembre 1912, personne n'est surpris d'entendre que les forces navales de l'Empire seront renforcées grâce à l'aide du Canada $^{58}$. L'important est de savoir la forme exacte que cette aide revêtira. Entre-temps, on se perd en conjectures. La Patrie déclare que les Canadiens acceptent volontiers l'idée de la participation à la défense de l'Empire tandis que Le Devoir, s'y opposant, s'interroge sur la réaction possible des députés fédéraux. Considérant le groupe conservateur du Québec, Georges Pelletier, son correspondant parlementaire, affirme que «Monk et Lamarche apparaissent comme à peu près les seuls récalcitrants», mais ils sont malades ${ }^{59}$. De Sévigny, les nationalistes n'attendent plus rien ${ }^{60}$. Et ils ont raison.

Dans les jours qui suivent la lecture du discours du Trône, Borden se charge de convaincre irrévocablement Sévigny qui le

55 L'Événement, 19 novembre $1912,8$.

56 Le Soleil, 15 novembre $1912,4$.

57 James G. Foley, op. cit., 13: le 19 novembre, Coderre triomphe par 2273 voix de majorité: il faut préciser aussi qu'Hochelaga n'est pas une circonscription typique du Québec puisqu'on y retrouve $38 \%$ d'anglophones, dont le bastion impérialiste de Westmount: voir John English, The Decline of Politics: The Conservatives and the Party System, 19011920 (Toronto et Buffalo, University of Toronto Press, [c 1977]), 81.

58 Les Débats, session 1912-1913, vol. I:2.

59 La Patrie, 22 novembre 1912, 4; Le Devoir, 25 novembre 1912, 1. Cependant, l'avenir lui donnera tort, mais de peu.

60 Ibid., 20 novembre 1912, 1. 
rencontre le 26 novembre ${ }^{61}$. Puis, dans l'après-midi du 27 novembre, Albert Sévigny, mis au courant des intentions exactes de Borden, annonce officiellement à son chef qu'il soutiendra son projet d'aide aux forces navales de l'Empire. Dans son journal personnel, Borden le précise nettement: "Met French Members this afternoon. Told them we propose repealing Laurier Naval bill. Gave them an outline of our permanent policy. Boulay, Barette [sic], Bellemare, Achim \& Guilbault said that agreed that proposals are wise but they are bound by promises. Lavallée \& Gauthier said notwithstanding promises they wd vote for us. Also Paquet, Rainville, Blondin \& Sévigny. ${ }^{62}$ Ainsi, Albert Sévigny, répudiant toutes ses paroles et ses promesses des années 1910-1911, acceptera de voter en faveur de la contribution d'urgence que s'apprête à dévoiler Borden. Il semble bien que c'en est fait, désormais, des votes contre son parti et de l'appui conditionnel qui avaient caractérisé son attitude depuis le début de 1912.

Le 5 décembre 1912, le premier ministre dépose en Chambre le projet de loi «autorisant certaines mesures à l'effet d'augmenter les forces navales de l'empire [sic]». \$35000 000 sont offerts à la Grande-Bretagne pour construire trois vaisseaux de type dreadnought et devant servir à la défense de l'Empire. Construits en Angleterre et faisant partie de la marine britannique, ils seront entretenus par cette dernière jusqu'au moment où ils pourraient faire partie d'une unité canadienne de la marine britannique ${ }^{63}$. En présentant ce projet de loi, Borden «set [alors] in motion one of the longest, most implacable, and most famous debates since Confederation ${ }^{64} \gg$. Jusqu'au 15 mai 1913, en effet, les discussions en Chambre s'avéreront aussi interminables que passionnées et virulentes.

Les libéraux, comme la minorité des «conservateurs-nationalistes» cités plus haut par Borden, proposent, lors des lectures des résolutions et du projet lui-même, une série d'amendements tous refusés par le gouvernement. Sévigny, comme prévu, appuie son chef à chaque occasion ${ }^{65}$. Croyant probablement à l'éloquence de

61 APC, Fonds Borden, Privés, Journal personnel, 26 novembre 1912; les arguments de Borden qui auraient pu aussi convaincre Sévigny et les autres sont, entre autres: 1L'abrogation de la loi navale de Laurier; 2- L'importance de la représentation canadienne au Comité de la Défense impériale; 3- La possibilité de développer au Québec une solide industrie de construction navale lors de la mise en application d'une future loi navale permanente: Robert Craig Brown, Robert Laird Borden. A Biography..., 238.

62 APC, Fonds Borden, Privés, Journal personnel, 27 novembre 1912.

63 Les Débats, session 1912-1913, vol. I: 687-706.

64 Gilbert Norman Tucker, The Naval Service..., t. I: 188. Il est bon de remarquer que Borden ne révoque pas la loi Laurier de 1910.

65 Voir Les Débats, session 1912-1913, vol. I à V; pour la description de tous ces événements, voir Heath Macquarrie, ed., Robert Laird Borden..., 188-197. 
ses votes, le député de Dorchester se tait au cours de ces discussions. Jamais, même si ses adversaires le lui demandent, il n'émet les raisons fondamentales de son changement d'attitude sur la question navale, se contentant plutôt d'affirmer que «cela viendra en temps et lieu ${ }^{66}$. Ce n'est qu'en comité plénier précédant la troisième lecture, alors que les libéraux décident de passer à l'obstruction systématique, que Sévigny se résout à prendre la parole, mais seulement pour interrompre leurs orateurs désireux de prolonger le débat. Ainsi interrompus, cependant, les députés libéraux en profitent pour rappeler à Sévigny ses paroles dans DrummondArthabaska et lors de l'élection de 1911, alors qu'il fustigeait autant la politique de Borden que celle de Laurier. Aussi promptement, le député de Dorchester nie les avoir prononcées et réplique même, avec une assurance déconcertante, qu'il était représentant du parti conservateur en 1911, qu'il était avec son "chef, M. Borden, qui était opposé à votre marine»; il ajoute avoir déjà déclaré qu'il serait contre Borden si ce dernier préconisait de créer «une grosse marine et en plus donner 25 millions par année à l'Angleterre [...], mais comme ce n'est pas là sa politique», il l'appuie aujourd'hui; enfin, Sévigny affirme qu'il est "prêt demain même, à répéter dans la province de Québec tout ce qu' [il a] dit [auparavant] sur cette question navale ${ }^{67} \gg$. Le 15 mai 1913 , le projet Borden est finalement adopté en troisième lecture et le député de Dorchester, sans hésitation, l'approuve. Albert Sévigny pose alors un geste, incompréhensible aux yeux de certains, mais combien rémunérateur pour sa carrière dans le parti ${ }^{68}$.

Ses adversaires, particulièrement les adeptes du mouvement nationaliste, ne manquent pas de le répudier comme ils l'ont fait au cours des discussions du projet Borden. Pour eux, Sévigny est peut-être celui de ce "'groupe de vendu' [...] qui a le plus de front ${ }^{69} \gg$. Â leurs yeux, le député de Dorchester renie tout un passé pour obtenir tout simplement les faveurs de Borden et le poste de solliciteur général ${ }^{70}$. S'il était en faveur de la contribution Borden en 1910 et 1911, écrit Le Devoir, «pourquoi faisait-il [alors] cause

66 Les Débats, session 1912-1913, vol. III: 4850.

67 Voir Ibid., 4995-5000; Ibid., vol. V: 9563-9564.

68 Ibid., 10349-10350. Le projet de loi Borden, accepté par la Chambre des communes, après que le gouvernement eut imposé une limite de temps aux débats, est cependant rejeté, en fin mai 1913, par le Sénat composé d'un nombre majoritaire de libéraux. En janvier 1914, la question navale reviendra devant la Chambre des communes grâce à DavidOvide Lespérance, député conservateur de Montmagny. Il dépose, le 27 janvier 1914, un projet de loi tendant à abroger la loi navale de Laurier, et c'est Paul-Émile Lamarche qui l'appuie. Cependant, on n'en entend plus parler par la suite: voir Ibid., session 1914, vol. I: 212.

69 Le Nationaliste, 1er décembre 1912, 1.

70 Le Devoir, 21 novembre 1912, 1. 
commune avec MM. Bourassa et Lavergne qui la dénonçaient» ${ }^{71}$. Confronté ainsi au passé, Sévigny risque de perdre la face.

Mais alors, pourquoi donc a-t-il choisi en 1912-1913 de suivre Borden et son projet de contribution? Sévigny lui-même s'explique d'abord au cours de l'été de 1913 alors qu'il parcourt sa circonscription, puis plus particulièrement lors d'une polémique qu'il engage sur ce sujet avec Omer Héroux du Devoir à l'automne de 1913. Sévigny écrit alors à Héroux:

Je dois vous dire que j'ai voté pour la contribution parce que ma conscience et mon jugement m'ont dit que c'était mon devoir de le faire dans l'intérêt du Canada et de l'Empire dont nous faisons partie [...]. Vous vous prétendez conscient de votre dignité de sujet britannique et soucieux de l'avenir de votre pays; c'est parce que j'ai la même prétention que j'ai cru bien faire en votant pour la contribution.

Dites ce que vous voudrez de mes discours passés ou présents, mais croyez bien que cela ne m'empêchera pas de parler en faveur de la contribution [...]. Je considère les arguments en faveur de la contribution plus forts que les vôtres contre [...].

J'ai voté pour la contribution parce que le Canada fait partie de l'Empire britannique et parce que je crois que nous avons besoin de la suprématie de la marine anglaise pour le Canada comme pour le reste de l'Empire. La marine anglaise ne nous a jamais coûté un sou, ni un homme, ni une goutte de sang. Trouvez-moi un seul pays qui a vécu en paix comme le Canada depuis cent ans. Et si vous me répondez que nous ne devons pas cette paix à la puissance de l'Angleterre, nous ne sommes pas d'accord, mais je crois sincèrement que cette puissance est d'importance vitale pour l'avenir de notre pays et c'est à l'avenir qu'il faut songer [...].

Je sais que vous allez me répondre en alléguant la question de principe et sur cette question je ne m'accorde avec vous tant qu'il me faudra dire que le Canada fait partie de l'Empire Britannique et que cet Empire doit être respecté par ses ennemis $[\ldots]$.

Pour sauver sa peau, c'est mieux de dire non, mais pour faire son devoir, c'est mieux de dire oui [...]. Vos beaux cris peuvent nourrir certaines passions, mais j'aime mieux la raison, surtout depuis que j'ai connu le très honorable R.L. Borden qui a toute ma confiance.

La «position» que j'ai prise avec lui s'accorde avec mon honneur, mon intelligence et ma conscience. ${ }^{72}$

71 Ibid., 10 mars 1913, 1.

72 Ibid., 24 septembre 1913, 1; Ibid., 2 octobre 1913, 2. 
Sincère ou non, le député de Dorchester ne parvient pas à convaincre. Comme le lui souligne Omer Héroux, «ce n'est pas d'hier que le Canada fait partie de l'Empire britannique» et sa suprématie devait être aussi importante pour le Canada lors de Drummond-Arthabaska et de l'élection de 1911 qu'elle l'est alors $^{73}$. À partir de mai 1913, Albert Sévigny commence la longue et épuisante tentative de persuader le Québec de sa sincérité sur la question de la coopération navale avec l'Empire. Ce ne sera pas chose facile.

Albert Sévigny laisse donc supposer qu'il n'a pu résister, sur cette question navale, aux tiraillements qui l'avaient assailli lors de l'affaire du Keewatin. Cette fois, il est des dix conservateursnationalistes qui ne reconnaissent plus les principes à la base même de leurs discours des années 1910-1911 et qui appuient le gouvernement Borden. Le plébiscite sur la question navale, la défense de l'autonomie du Canada face à l'Angleterre ainsi que l'indépendance face aux partis politiques apparaissent ici bien écartés. Selon Henri Bourassa, le blâme en revient aux ministres québécois. Si ces ministres, écrira-t-il, «avaient fortifié le groupe de députés élus à la même enseigne qu'eux, au lieu de les désorganiser et d'en démoraliser la moitié ou les trois-quarts par les menaces, les flatteries ou la corruption, ils n'auraient pas été obligés d'en venir à la dernière extrémité» ${ }^{34}$. Bourassa, cependant, aurait dû se demander si ces jeunes députés auraient cédé aussi facilement si lui-même avait décidé de se présenter en 1911 et de les encadrer à Ottawa; près d'eux, et influent, n'aurait-il pas réussi à enrayer l'action possible de ces ministres et, conséquemment, neutraliser ce que le système de partis a pu leur présenter de gratifiant et de sécurisant?

Car c'est de cela qu'il s'agit aussi. Au-delà d'une approbation de la politique de Borden fondée sur "sa conscience et son devoir», c'est également le parti conservateur au pouvoir, en possession du patronage, des titres et fonctions à décerner tout comme d'une discipline à faire respecter, qui a raison de la ténacité et de l'orientation qu'Albert Sévigny avait privilégiée depuis les débuts de 1912. S'il appert que, désormais, le député de Dorchester acquiescera

73 Ibid., 2 octobre 1913, 1.

74 Ibid., 5 juin 1913, 1; par ailleurs, nous croyons, à l'instar de Joseph Levitt et au contraire de Robert Rumilly et d'Elizabeth H. Armstrong, que ce n'est pas l'influence du mouvement nationaliste [et conséquemment des 7 conservateurs-nationalistes fidèles] qui a fait battre le projet au Sénat. C'est plutôt l'impuissance des deux partis à s'entendre sur un compromis acceptable quant à l'aide navale à octroyer à l'Empire dont ils reconnaissent tous deux l'importance: Joseph Levitt, "La perspective nationaliste d'Henri Bourassa", Revue d'Histoire de l'Amérique française, XXII, 4 (mars 1969): 580; Robert Rumilly, Histoire de la province de Québec (Montréal, Montréal-Éditions, s.d.), t. XVIII: 50; Elizabeth H. Armstrong, The Crisis of Quebec, 1914-1918 (New York, Columbia University Press, 1937), 34. 
sans discernement à toute politique préconisée par son parti, il faut en voir là, selon nous, l'une des raisons principales. En somme, après une année et demie passée à Ottawa, loin des préoccupations des temps d'opposition où le parti conservateur avait bien peu à offrir pour garder ses troupes dans le rang, Albert Sévigny, qui apprend vite, a choisi. Il a renoncé aux principes nationalistes défendus par Henri Bourassa et s'est associé étroitement à la politique de son parti dans l'espoir de s'assurer une carrière plus rapide.

\section{Conclusion}

Dans les années qui suivent, cette attitude d'Albert Sévigny ne fera que s'accentuer atteignant un paroxysme au moment des grands débats sur la participation canadienne à la Grande Guerre. De 1913 à 1918, le député de Dorchester appuiera constamment, inconditionnellement, les politiques mises de l'avant par Borden. On l'identifiera à «l'un des plus actifs et des plus éloquents lieutenants [du premier ministre] ${ }^{75}$. En 1917, devenu ministre, il ira même à l'encontre des sentiments de la grande majorité des Canadiens français et supportera le projet de conscription militaire proposé par son chef. Avec seulement deux autres ex-conservateursnationalistes, Sévigny formera alors, aux dires mêmes des disciples de Bourassa, le «triumvirat des traîtres» à la nation. Qui plus est, à la fin de cette même année, il acceptera de devenir pratiquement le seul ministre francophone du gouvernement d'Union formé par Borden pour appliquer la conscription ${ }^{76}$. Dans ces circonstances, il est facile de constater qu'en 1913, Albert Sévigny avait bel et bien enterré ses idées conservatrices-nationalistes. De toute façon, la population se chargera de le lui rappeler en le chassant pour toujours de la vie politique lors des élections générales de décembre 1917. Il démissionnera le 7 mars 1918.

À en juger par la conduite d'Albert Sévigny, il faut conclure que la victoire tory de 1911 et ses suites ont considérablement modifié l'attitude du groupe conservateur-nationaliste face au parti de Borden et au système de partis canadien. Ce comportement d'après 1911 ne fut cependant ni homogène ni constitué d'un bloc sans fissure: plusieurs tendances s'y retrouvèrent entremêlées, souvent inextricables. Albert Sévigny, pour sa part, s'est engagé progressivement dans la voie jugée la plus dégradante par Henri Bourassa et ses disciples.

Plusieurs facteurs peuvent expliquer ces choix d'Albert Sévigny et les uns n'excluent pas nécessairement les autres. Malgré

75 L'Événement, 21 février 1914, 1.

76 L'autre, Pierre-Édouard Blondin, s'est enrôlé et a quitté le Canada. 
ses dénégations répétées au nom de sa conscience, de son sens du devoir et du sacrifice, il est indéniable que l'opportunisme et l'ambition ont influencé plusieurs des gestes de ce député. Par ailleurs, l'intransigeance et les gratifications du système de partis ont aussi eu raison de lui; ceci le conduira d'ailleurs à une identification à son parti et à une loyauté à son chef plus forte chez lui que chez certains de ses collègues. En outre, il nous apparaît pertinent de souligner que Sévigny a pu évoluer dans sa perception du nationalisme canadien. Après avoir épousé celui de Henri Bourassa, il se pourrait bien qu'il se soit rallié, par la suite, par conviction et en raison d'une meilleure connaissance du Canada, à cet autre type de nationalisme - identifié par certains de pur impérialisme - qui privilégie un rôle accru du Canada dans l'Empire afin de maximiser au plus haut degré son développement global. Conséquent avec lui-même, Sévigny se joindrait progressivement à Borden, ardent défenseur de ce nationalisme.

Au-delà du cas Sévigny, il faut noter la dure réalité de l'évolution des conservateurs-nationalistes après les élections de 1911. Sauf un, en effet, tous se sont progressivement ralliés à Borden ${ }^{77}$. À compter de 1913, le groupe lui-même n'existait déjà plus. À la longue, ils furent si bien absorbés par le système de partis que plus personne ne les perçut comme conservateurs-nationalistes, pas même au moment de la conscription de 1917 lorsque huit d'entre ceux encore à Ottawa prirent des positions qui rappelaient 1911 . Cette situation laisse perplexe mais fait bien voir l'implacable réalité de la vie parlementaire.

À une échelle plus large, cette évolution n'est pas sans analogie avec d'autres analyses politiques comme celles touchant au Fragment Party. À ce sujet, le politicologue John McMeneny a démontré par exemple que les

Fragment parties exist as a result of conflict within the elite of a parliamentary party. The fragment party is established by an individual who leaves the elite of a parliamentary party after failing to sway it to his opinion in a given conflict. While the fragment party expresses a popular grievance, the party has no mass base of support. The fragment's leadership is out of touch with those groups whose support would give it sustaining power. Moreover, the elitist or aristocratic career of a fragment party leader does not equip him with the talents, connections, or perhaps even the inclination to develop a mass membership organization. Consequently, fragment parties are evanescent and the subsequent political careers of their leaders are often shortlived as well $[\ldots]$. The identity of the $[\ldots]$

77 Il s’agit de Paul-Émile Lamarche. 
fragment party is defined in terms of the founding or leading personality rather than in terms of popular group support.

Or, le groupe parlementaire des conservateurs-nationalistes réunit plusieurs de ces caractéristiques et est entiché des mêmes lacunes. Il donne l'image d'un Fragment Party, formé à la suite d'un conflit au sein de l'élite parlementaire du parti conservateur, dominé par quelques leaders qui prirent tôt leur distance, élitiste, sans organisation et, conséquemment, voué à une existence brève. De fait, on sait que Henri Bourassa n'a ni cherché à se faire élire en 1911, ni cherché à encadrer réellement le groupe par la suite. On constate aussi que F.D. Monk, malade, se retira à toute fin pratique de la politique après sa démission du Cabinet en 1912. De plus, ces leaders ne se préoccupèrent pas d'élargir la base électorale de 1911 qui aurait pu donner une armature au groupe et surveiller son action politique. Face à cela, on peut se demander si ce groupe parlementaire, ce "Fragment Party», n'était tout simplement pas appelé à se désagréger tôt conduisant ainsi plus facilement des députés comme Albert Sévigny dans le giron de Borden.

78 Conrad Winn et John McMeneny, Political Parties in Canada (Toronto, Montréal..., McGraw-Hill Ryerson Limited, [c 1976]), 30; cela rappelle bien aussi les «petits partis de personnalités») décrits par Maurice Duverger, Les Partis politiques (Paris, Librairie Armand Colin, 1976) (d'abord paru en 1951), 323-324. 